\title{
AGILE detection of GeV $\gamma$-ray emission from the SNR W28
}

\author{
A. Giuliani ${ }^{1}$, M. Tavani ${ }^{2,3}$, A. Bulgarelli ${ }^{5}$, E. Striani ${ }^{3,12}$, S. Sabatini ${ }^{2}$, M. Cardillo ${ }^{2}$, Y. Fukui ${ }^{7}$, A. Kawamura ${ }^{7}$,

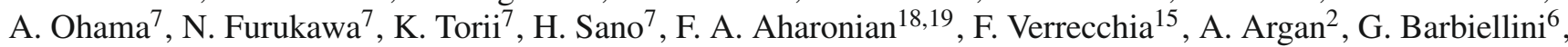
P. A. Caraveo ${ }^{1}$, P. W. Cattaneo ${ }^{8}$, A. W. Chen ${ }^{1}$, V. Cocco $^{12}$, E. Costa ${ }^{2}$, F. D'Ammando ${ }^{2,3}$, E. Del Monte ${ }^{2}$, G. De Paris ${ }^{2}$, G. Di Cocco ${ }^{5}$, I. Donnarumma ${ }^{2}$, Y. Evangelista ${ }^{2}$, M. Feroci ${ }^{2}$, M. Fiorini ${ }^{1}$, T. Froysland ${ }^{3,4}$, F. Fuschino ${ }^{5}$, M. Galli ${ }^{10}$, F. Gianotti ${ }^{5}$, C. Labanti ${ }^{5}$, Y. Lapshov ${ }^{2}$, F. Lazzarotto ${ }^{2}$, P. Lipari ${ }^{11}$, F. Longo ${ }^{6}$, M. Marisaldi $^{5}$, S. Mereghetti $^{1}$,

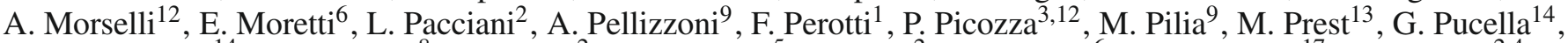
M. Rapisarda ${ }^{14}$, A. Rappoldi ${ }^{8}$, P. Soffitta $^{2}$, M. Trifoglio ${ }^{5}$, A. Trois ${ }^{2}$, E. Vallazza $^{6}$, S. Vercellone ${ }^{17}$, V. Vittorini' ${ }^{2,4}$, A. Zambra ${ }^{1}$, D. Zanello ${ }^{9}$, C. Pittori ${ }^{15}$, P. Santolamazza ${ }^{15}$, P. Giommi ${ }^{15}$, S. Colafrancesco ${ }^{15}$, and L. Salotti ${ }^{16}$

(Affiliations can be found after the references)

Received 13 February 2010 / Accepted 5 May 2010

\section{ABSTRACT}

\begin{abstract}
Aims. Supernova remnants (SNRs) are believed to be the main sources of Galactic cosmic rays. Molecular clouds associated with SNRs can produce gamma-ray emission by means of the interaction of accelerated particles with the concentrated gas. The middle-aged SNR W28, because of its associated system of dense molecular clouds, provides an excellent opportunity to test this hypothesis.

Methods. We present the AGILE/GRID observations of SNR W28, and compare them with observations at other wavelengths (TeV and ${ }^{12} \mathrm{CO}$ $(J=1 \rightarrow 0)$ molecular line emission).

Results. The gamma-ray flux detected by AGILE from the dominant source associated with W28 is $(14 \pm 5) \times 10^{-8} \mathrm{ph} \mathrm{cm}^{-2} \mathrm{~s}^{-1}$ for $E>400 \mathrm{MeV}$. This source is positionally well correlated with the $\mathrm{TeV}$ emission observed by the HESS telescope. The local variations in the GeV to TeV flux ratio imply that there is a difference between the CR spectra of the north-west and south molecular cloud complexes. A model based on a hadronicinduced interaction and diffusion with two molecular clouds at different distances from the W28 shell can explain both the morphological and spectral features observed by both AGILE in the MeV-GeV energy range and the HESS telescope in the TeV energy range. The combined set of AGILE and H.E.S.S. data strongly support a hadronic model for the gamma-ray production in W28.
\end{abstract}

Key words. acceleration of particles - diffusion - ISM: supernova remnants - gamma rays: ISM

\section{Introduction}

Known also as G6.4-0.1, SNR W28 is a middle-aged supernova remnant (with an age of at least 35 thousand years), located at a distance between 1.8 and $3.3 \mathrm{kpc}$ in the inner region of the Galaxy $(l, b=6.71,-0.05)$. It is a mixed-morphology SNR, of large (about 48 arcmin) angular size (see Green 2009). At radio wavelengths a shell structure, where the interaction of the SNR ejecta and the ISM creates a shock, is clearly visible (see Fig. 1). The spectral index between 328 and $1415 \mathrm{MHz}$ is $\alpha=-0.35 \pm 0.18$ and experiences large local variations that are correlated with the flux density (Dubner et al. 2000). The ${ }^{12} \mathrm{CO}$ $(J=1 \rightarrow 0)$ molecular line observations taken by the NANTEN telescope detect a system of molecular clouds associated with the SNR (Mizuno \& Fukui 2004). Contours in Fig. 1 show the intensity of the ${ }^{12} \mathrm{CO}$ line emission integrated over the velocity range 3 to $27 \mathrm{~km} \mathrm{~s}^{-1}$, corresponding to the dynamical distances compatible with W28. The emission appears to be concentrated in two main complexes close to the northwestern (cloud N) and southern (cloud S) part of the SNR. Evidence of interaction between the remnant and the system of molecular clouds is given by the detection of $1720 \mathrm{MHz} \mathrm{OH}$ maser emission (Frail et al. 1994) and the observation of an unusually high value of the ratio $\mathrm{CO}(J=3-2) /(J=2-1)$ (Arikawa et al. 1999).

Two TeV sources have been detected by the HESS Cherenkov telescope near W28 (Aharonian et al. 2006).
The positions of the sources HESS J1801-233 and HESS J1800240 (A, B, and C) are very well correlated with, respectively, the $\mathrm{N}$ and $\mathrm{S}$ molecular clouds resolved by the NANTEN telescope (Aharonian et al.( 2008)Akhperjanian\& Bazer-Bachi ). In the MeV-GeV energy range, a source compatible with W28 was reported in the Third EGRET catalog, 3EG J1800-2338 (Hartman et al., 1999). The flux measured by EGRET above $100 \mathrm{MeV}$ was $61.3 \pm 6.7 \mathrm{ph} \mathrm{cm}^{-2} \mathrm{~s}^{-1}$ with a photon spectral index of 2.10. In both the Fermi LAT Bright Source List (0FGL J1801.6-2327, see Abdo et al. 2009) and the First AGILE Catalog (1AGL J1803-2258, see Pittori et al. 2009), a gamma-ray source centered on the position of cloud $\mathrm{N}$ is reported.

In this Letter, we present the results of deep AGILE observation of 1AGL J1803-2258, which, at energies greater than 400 $\mathrm{MeV}$ has a shape that turns out to be incompatible with a single point source, and is remarkably well spatially correlated with both the molecular cloud system seen by NANTEN and the TeV sources detected by HESS.

\section{Data analysis}

The Gamma-Ray Image Detector (GRID) onboard AGILE satellite (Tavani et al. 2008) extensively observed the Galactic plane during the years 2007-2009 at energies greater than $100 \mathrm{MeV}$ with an angular resolution better than the previous 


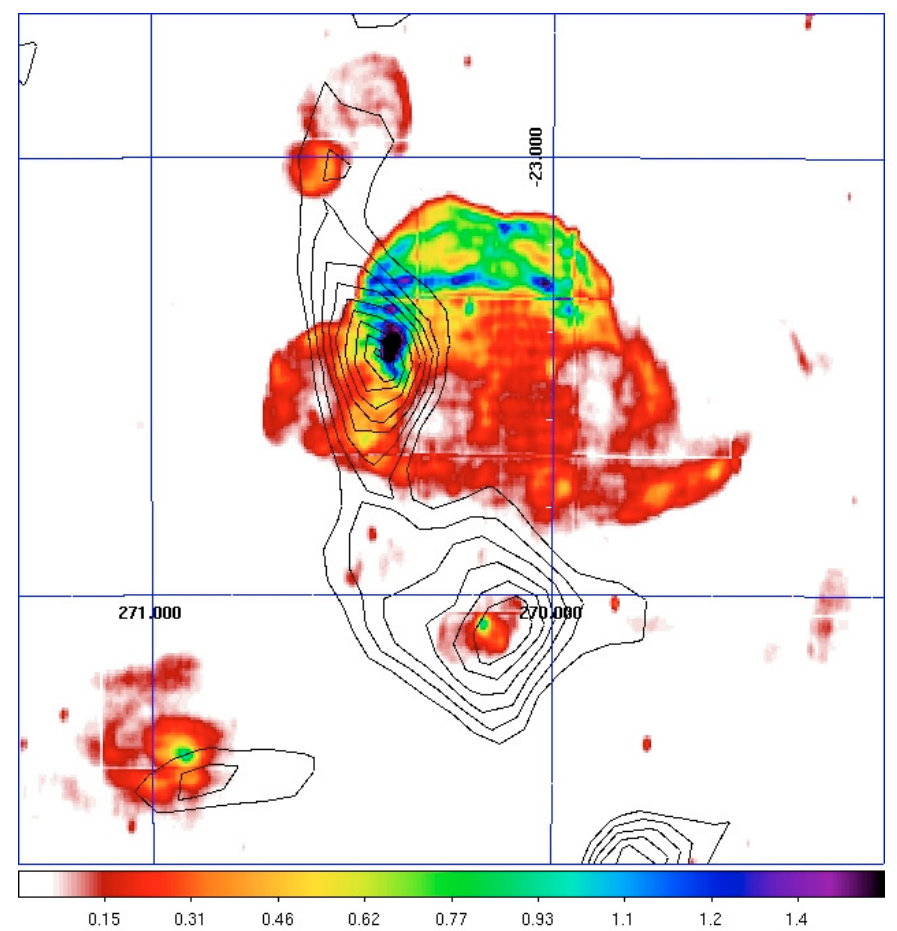

Fig. 1. Map of VLA $90 \mathrm{~cm}$ radio emission from SNR W28 in celestial J2000 coordinates RA and Dec. (Colours indicate the intensity in Jy beam ${ }^{-1}$ ). The black contours show the CO intensity emission obtained with the NANTEN radio telescope, which traces molecular clouds, integrated over the velocity interval of 3-27 km s${ }^{-1}$ (contours vary between 90 and $170 \mathrm{~K}$ deg with a step size of $10 \mathrm{~K} \mathrm{deg}$ ). Two concentrations of $\mathrm{CO}$ emission are clearly visible near the positions $\alpha, \delta=270.4,-23.4($ cloud $\mathrm{N}$ ) and $\alpha, \delta=270.2,-24.1$ (cloud S).

gamma-ray telescopes. Level-1 AGILE-GRID data were analyzed using the AGILE Standard Analysis Pipeline. The Anticoincidence System (ACS) and a set of hardware triggers performed the first reduction of the high rate of background events (charged particles and albedo gamma-rays) interacting with the instrument. A dedicated software processed the GRID and ACS signals and performed a reconstruction and selection of events in order to discriminate between background events and gamma-rays, deriving for the latter the energy and direction of the incoming photons. A simplified version of this software operated directly on board AGILE to reduce the telemetry throughput (Giuliani et al. 2006). A more complex version of the photon reconstruction and selection software was applied on the ground, producing a photon list containing arrival time, energy, and direction of every gamma-ray and the corresponding exposure history. Counts, exposure, and Galactic background gamma-ray maps, the last based on the Galactic diffuse emission model developed for AGILE (Giuliani et al. 2004), were created with a bin-size of $0.05 \times 0.05$ for photons with energy greater than $100 \mathrm{MeV}$ and $400 \mathrm{MeV}$. We selected only events flagged as confirmed gamma-ray events. All events collected during the South Atlantic Anomaly or whose reconstructed directions form angles with the satellite-Earth vector smaller than $90^{\circ}$ were rejected. To derive the average flux and spectrum of the source, we ran the AGILE point source analysis software ALIKE (Chen et al., in prep.), based on the maximum likelihood technique as described in Mattox et al. (1993), over the whole observing period. All the flux errors found by ALIKE (and reported in the following) take into account only the statistical uncertainities.

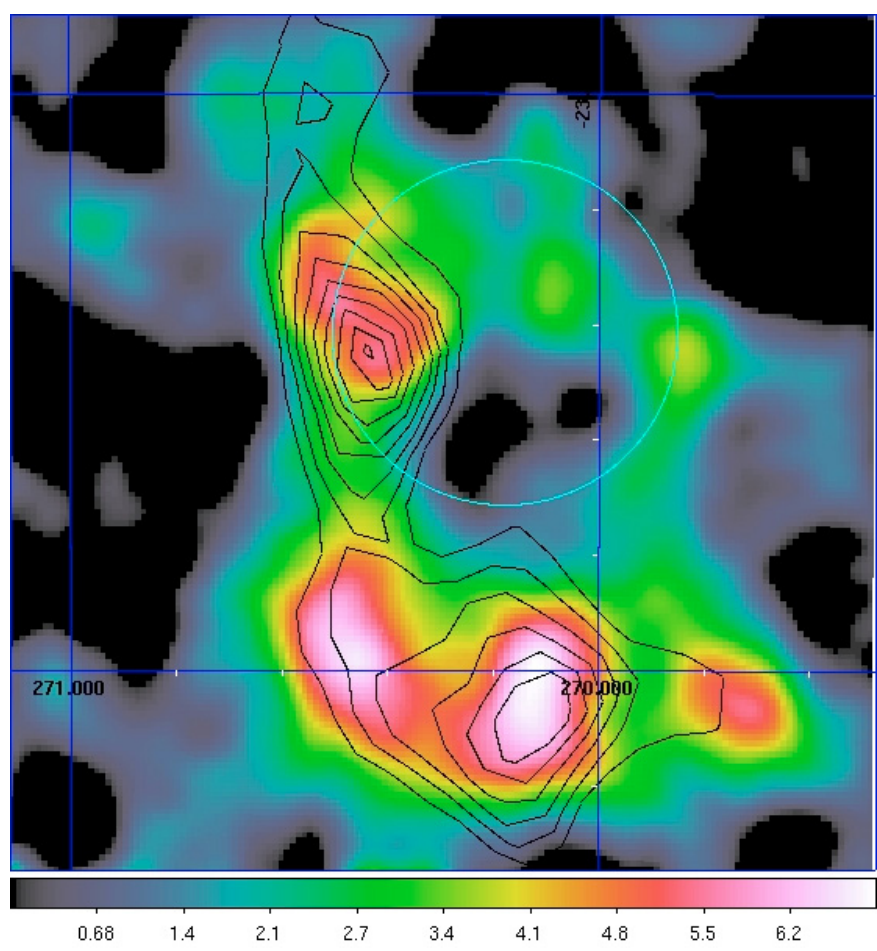

Fig. 2. Sky map in celestial J2000 coordinates, RA and Dec., of the significance $(\sigma)$ of the $\mathrm{TeV}$ emission detected by the HESS telescope (Aharonian et al.( 2008)Akhperjanian\& Bazer-Bachi ). The blue circle indicates the location of the supernova remnant W28. As in Fig. 1 the black contours show the $\mathrm{CO}$ intensity emission.

We estimated that the systematic errors are on the level of $10 \%$ of the reported fluxes.

\subsection{Results}

A prominent gamma-ray source associable with W28 (1AGL $\mathrm{J} 1803-2258)$ is clearly detected at a significance level of $7 \sigma$ with an average flux of $(40 \pm 11) \times 10^{-8} \mathrm{ph} \mathrm{cm}^{-2} \mathrm{~s}^{-1}$ for $E>100 \mathrm{MeV}$ above the prediction of the AGILE diffuse emission model. The positional analysis gives an elliptical error-box centered on $l=6.0^{\circ} b=-0.2^{\circ}$ with a mean radius of about $0.1^{\circ}$ consistent with both the EGRET and Fermi sources detected in the same region.

Figure 3 shows the counts map for the source 1AGL J18032258 at energies greater than $400 \mathrm{MeV}$. At these energies, the high angular resolution of the GRID detector allows us to perform a morphological analysis of the source, which shows a remarkable correspondence with the $\mathrm{TeV}$ emission observed in the same region. The total flux of the source at energies greater than $400 \mathrm{MeV}$ is $(14 \pm 5) \times 10^{-8} \mathrm{ph} \mathrm{cm}^{-2} \mathrm{~s}^{-1}$. Most of the emission is concentrated in a region coincident with the molecular cloud $\mathrm{N}$ (and the TeV source HESS J1801-233). A weaker gamma-ray diffuse emission appears to be superimposed on the molecular cloud $\mathrm{S}$ (and the TeV source complex HESS J1800-240). Performing a likelihood analysis with two point sources fixed at the locations of source $\mathrm{N}$ and source $\mathrm{S}$, we measured flux values $F_{\mathrm{N}}=(10 \pm 3) \times 10^{-8} \mathrm{ph} \mathrm{cm}^{-2} \mathrm{~s}^{-1}$ and $F_{\mathrm{S}}=(4 \pm 2) \times 10^{-8} \mathrm{ph} \mathrm{cm}^{-2} \mathrm{~s}^{-1}$ above $400 \mathrm{MeV}$. The two regions are not clearly resolved in the $100-400 \mathrm{MeV}$ energy range. Nevertheless, we performed a likelihood analysis assuming the same two point sources as in the analysis for the $E>400 \mathrm{MeV}$ energy range. This yielded a flux of $(30 \pm 6) \times 10^{-8} \mathrm{ph} \mathrm{cm}^{-2} \mathrm{~s}^{-1}$ 


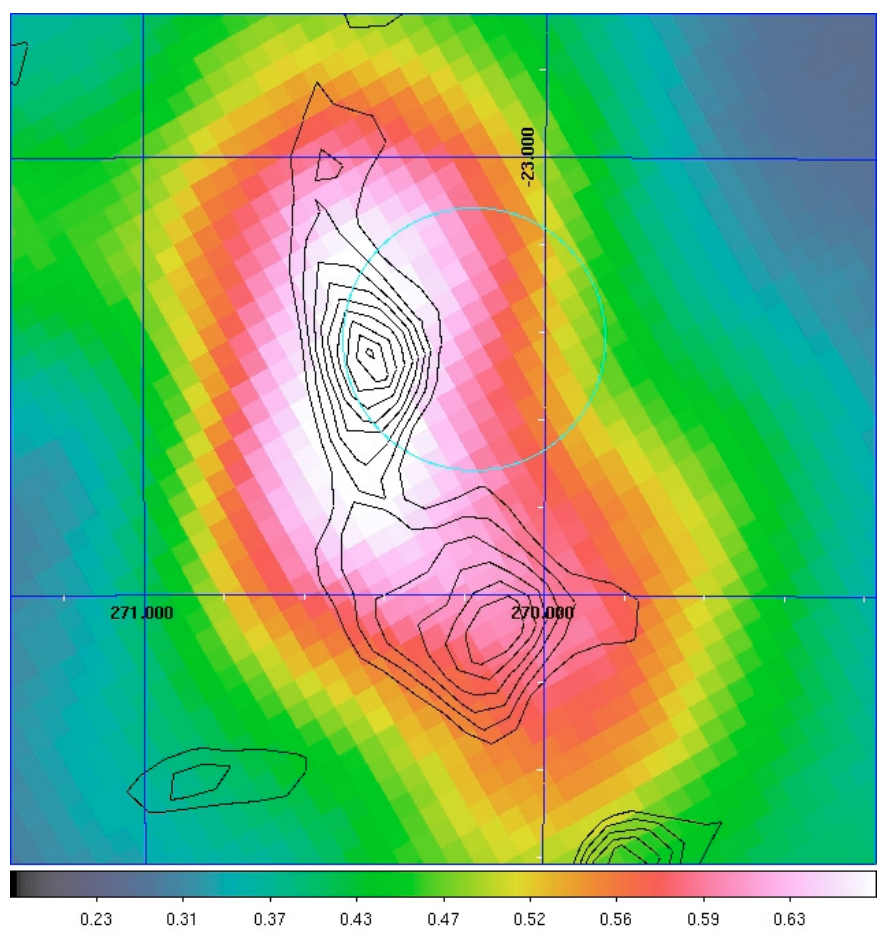

Fig. 3. Gaussian-smoothed AGILE counts map in Galactic coordinates for the W28 region (counts bin' ${ }^{-1}$ ). Only photons of energy greater than $400 \mathrm{MeV}$ have been used. The blue circle indicates the radio location of the supernova remnant W28. As in Fig. 1, the black contours indicate the $\mathrm{CO}$ intensity emission.

for cloud $\mathrm{N}$ and a $2 \sigma$ upper limit of $10 \times 10^{-8} \mathrm{ph} \mathrm{cm}^{-2} \mathrm{~s}^{-1}$ for cloud $\mathrm{S}$.

\section{Discussion}

Figure 4 shows the spectra for cloud N (black) and cloud S (red) in both the GRID and the HESS energy bands. While in the TeV energy band the complex of the source HESS 1800-240 is a factor of two brighter than the source HESS 1801-233, in the GeV band the reverse is true. Within a hadronic scenario, and assuming CRs are accelerated by W28 and then escape, the difference between the $\mathrm{TeV}$ to $\mathrm{GeV}$ flux ratios determined at the locations $\mathrm{N}$ and $\mathrm{S}$ implies that the two clouds have different proton spectra. This is not surprising because, for a turbulent medium, the diffusion coefficient of charged particles is expected to depend on the particle kinetic energy, $D=D(E)$. As a consequence, in the case of recent acceleration (within the past $10^{5}$ years), the proton spectrum depends strongly on the distance from the accelerator itself (Aharonian \& Atoyan 1996, Gabici et al. 2009). Hence the difference between the gamma-ray spectra of cloud $\mathrm{N}$ and $\mathrm{S}$ can be explained by assuming different distances between the clouds and the SNR. In the following, we show how this model can explain the $\mathrm{GeV} / \mathrm{TeV}$ spectra of the clouds assuming a set of parameters (shown in Table 1) that are compatible with other observational constraints. We estimated (using the mathematical formalism developed in Aharonian \& Atoyan 1996) the evolution of the protons and nuclei spectra diffusing in the interstellar medium at different distances from the supernova remnant. We assumed a distance of $2 \mathrm{kpc}$ and SNR age of 45000 years and that the particles have been injected continuously during the first 10000 years of its life, with a power-law energy spectrum of

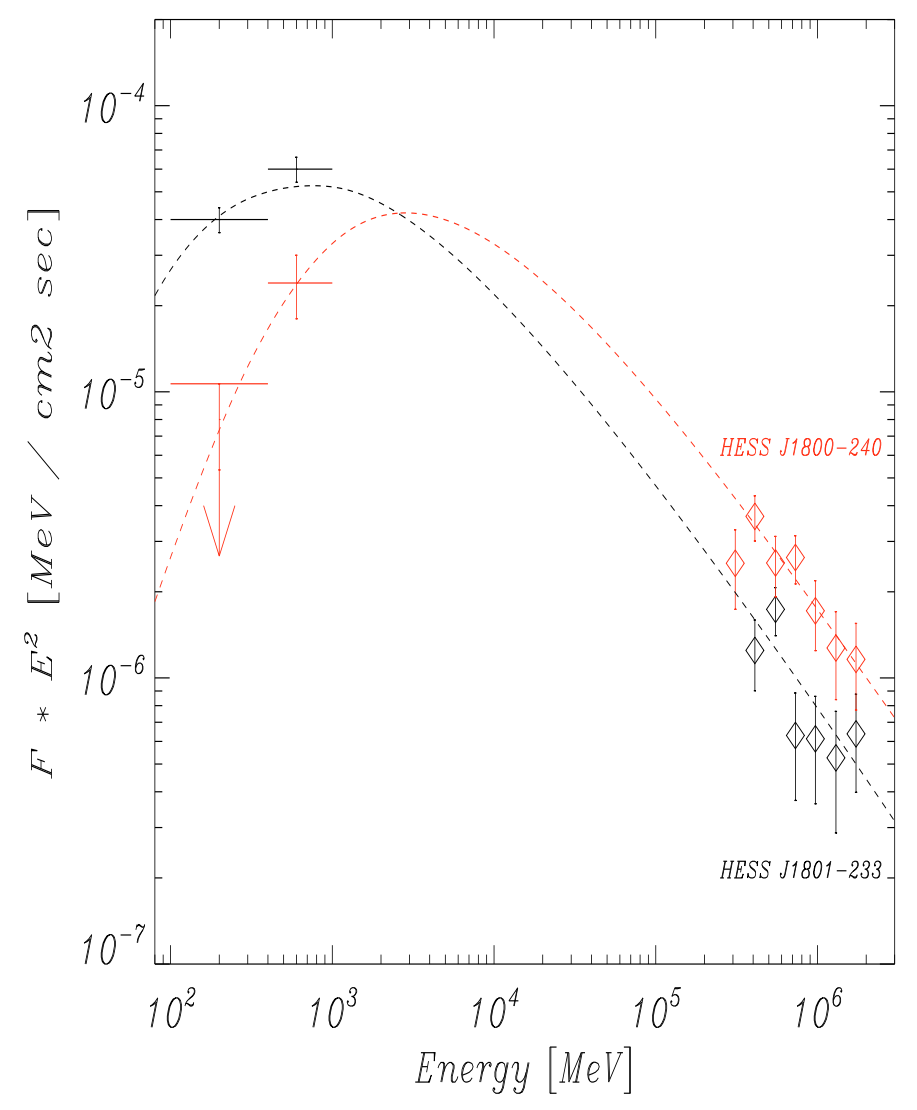

Fig. 4. Gamma-ray photon spectrum for W28 (AGILE points: $1 \sigma$ statistical errors, HESS points are from Aharonian et al. 2008). The curves represent the gamma-ray spectra estimated, accordling to the model presented in the text, for the cloud $\mathrm{N}$ (black) and the cloud $\mathrm{S}$ (red) assumed at a distance of 4 and $9 \mathrm{pc}$, respectively, from the particle acceleration site.

index 2.2. After the injection, we assumed that they propagate in the interstellar medium with a diffusion coefficient given by

$D=D_{0}\left(\frac{E}{10 \mathrm{GeV}}\right)^{\delta}$

where $\delta=0.5$ and we assume the rather slow diffusion coefficient $D_{0}=10^{26} \mathrm{~cm}^{2} \mathrm{~s}^{-1}$ inferred by Aharonian and Atoyan (1996) for a dense medium such as that of W28.

In Fig. 4, we compare the results of this model with the AGILE and HESS spectral data. The dashed lines correspond to a cloud accelerator distance of 4 (in black) and 9 (in red) parsecs. We note that a different set of parameters, the clouddistances and the SNR age, can lead to similar results (in general, any set of these parameters with the same ratio of distances to $\sqrt{\text { SNR age }}$ can reproduce the curves shown in Fig. 4). We choose here the distance of the cloud $\mathrm{S}$ to be the minimum distance compatible with the observations. A distance of $4 \mathrm{pc}$ for cloud $\mathrm{N}$, which requires assuming a projection effect of a few parsecs, and an age of 45000 years for W28 are then required to reproduce the $\mathrm{GeV}$ to $\mathrm{TeV}$ ratio of the clouds. We set the mass of the cloud $\mathrm{S}$ to be higher (by a factor 2.25) than that of cloud N, as can be inferred from the TeV fluxes and from the proton density predicted by this model at 4 and 9 pc from the SNR shell. The distances used in this model are consistent with the position of the clouds as observed in the radio maps (assuming an EarthSNR distance of $2 \mathrm{kpc}, 10$ parsecs corresponds to $0.29 \mathrm{deg}$ ), taking into account posible projection effects. Assuming a mass 
Table 1. Parameters of the model used to fit the AGILE and HESS spectral data, see Fig. 4.

\begin{tabular}{ll}
\hline \hline SNR age & $45000 \mathrm{yr}$ \\
SNR distance & $2 \mathrm{kpc}$ \\
CR total energy & $3.3 \times 10^{49} \mathrm{erg}$ \\
CR Injection index & 2.2 \\
Diffusion coeff. @ $10 \mathrm{GeV}\left(D_{0}\right)$ & $10^{26} \mathrm{~cm}^{2} \mathrm{~s}^{-1}$ \\
Diffusion coeff. spectral index $(\delta)$ & 0.5 \\
Cloud N (mass, distance from the W28 shell) & $2.0 \times 10^{4} M_{\odot}, 4 \mathrm{pc}$ \\
Cloud S (mass, distance from the W28 shell) & $4.5 \times 10^{4} M_{\odot}, 9 \mathrm{pc}$
\end{tabular}

for the two molecular clouds, it is possible to evaluate the total energy carried by the protons accelerated by W28. We used $2.0 \times 10^{4} M_{\odot}$ for cloud $\mathrm{N}$ and $4.5 \times 10^{4} M_{\odot}$ for cloud $\mathrm{S}$, in agreement with the estimate based on NANTEN data given in Aharonian et al.( 2008)Akhperjanian\& Bazer-Bachi . This leads to a proton total energy of $3.3 \times 10^{49} \mathrm{erg}$, a value in good agreement with the CR energy release from a SNR expected by theoretical considerations.

Finally, we briefly consider a leptonic scenario for the gamma-ray emission from W28. Relativistic electrons can produce gamma rays by means of inverse Compton (IC) and/or bremsstrahlung in a dense target. The cooling times for these processes are

$$
\begin{aligned}
t_{\mathrm{br}} & =4 \times 10^{7}\left(\frac{1 \mathrm{~cm}^{-3}}{n}\right) \mathrm{yr} \\
t_{\mathrm{IC}}(E) & =3 \times 10^{8}\left(\frac{1 \mathrm{eV} \mathrm{cm}^{-3}}{w_{r}}\right)\left(\frac{1 \mathrm{GeV}}{E}\right) \mathrm{yr}
\end{aligned}
$$

where $n$ is the gas density and $w_{r}$ is the energy density of the radiation field (note that Eq. (3) refers to IC scattering in the Thompson regime). For comparison, the cooling time for protons producing gamma rays by proton-proton interactions is

$t_{\mathrm{pp}}=1.6 \times 10^{8}\left(\frac{1 \mathrm{~cm}^{-3}}{n}\right) \mathrm{yr}$.

Inverse Compton is disfavoured because the strong correlation detected (especially at $\mathrm{TeV}$ energies) between the gamma-ray intensity and dense gas is not expected for evolved SNR such as W28. In principle, bremsstrahlung can produce the observed gamma-ray emission. Assuming that TeV gamma-rays are produced by $\sim 10 \mathrm{TeV}$ electrons, bremsstrahlung dominates over the IC in very dense regions $\left(n>1000 \mathrm{~cm}^{-3}\right)$. However, these energetic electrons are expected to be produced only at the early epochs of the SNR (Gabici et al. 2009), and can only marginally survive for the age of this source because of strong synchrotron and IC cooling. Furthermore, to explain the observed fluxes, a leptonic model would require a very large e/p ratio in accelerated particles (larger than 0.25, comparing Eqs. (2) and (4)), while a ratio near 0.01 is expected from the CR measurements.

\section{Conclusions}

We have analyzed the AGILE deep observations of SNR W28 to determine the existence of several contributions to the gammaray emission from this SNR. A highly significant gamma-ray source $(7 \sigma$ for $E>100 \mathrm{MeV}$ ) is associated with W28. This source has an average flux of $(40 \pm 11) \times 10^{-8} \mathrm{ph} \mathrm{cm}^{-2} \mathrm{~s}^{-1}$ during the period covered by the AGILE observations. For photon energies above $400 \mathrm{MeV}$, AGILE also detected a SE extension corresponding to a massive molecular cloud. We have proposed a model based on a hadronic-induced interaction with two molecular clouds adjacent to the SNR to fit the observations. This model explains the morphological and spectral features detected by both AGILE in the MeV-GeV energy range and the HESS telescope in the TeV energy range. Setting the distances and masses of the two main molecular clouds to values compatible with the radio $\mathrm{CO}$ observations, we hve been to estimate that the total energy in protons is $3.3 \times 10^{49} \mathrm{erg}$. Our data and analysis provide strong support to a hadronic origin of the gamma-ray emission from W28.

Acknowledgements. The AGILE Mission is funded by the Italian Space Agency (ASI) with scientific and programmatic participation by the Italian Institute of Astrophysics (INAF) and the Italian Institute of Nuclear Physics (INFN).Investigation carried out with partial support by the ASI contract no. I/089//06/2.

\section{References}

Abdo, A. A., Ackermann, M., Ajello, M., et al. 2009, ApJS, 183, 46 Aharonian, F. A., \& Atoyan, A. M. 1996, A\&A, 309, 917

Aharonian, F., Akhperjanian, A. G., Bazer-Bachi, A. R., et al. 2006, ApJ, 636, 777

Aharonian, F., Akhperjanian, A. G., Bazer-Bachi, A. R., et al. 2008, A\&A, 481, 401

Arikawa, Y., Tatematsu, K., Sekimoto, Y., et al. 1999, PASJ, 51, L7

Dubner, G. M., Velázquez, P. F., Goss, W. M., et al. 2000, AJ, 120, 1933 Frail, D. A., Goss, W. M., \& Slysh, V. I. 1994, ApJ, 424, L111

Gabici, S., Aharonian, F. A., \& Casanova, S. 2009, MNRAS, 396, 1629

Giuliani, A., Chen, A., Mereghetti, S., et al. 2004, Mem. S.A.It. Sup., 5, 135

Giuliani, A., Cocco, V., Mereghetti, S., et al. 2006, Nucl. Instr. Meth. A, 568, 692

Green, D. A. 2009, Bull. Astron. Soc. India, 37, 45

Hartman, R. C., Bertsch, D. L., Bloom, S. D., et al. 1999, ApJS, 123, 79

Mattox, J. R., Bertsch, D. L., Chiang, J., et al. 1993, ApJ, 410, 609

Mizuno, A., \& Fukui, Y. 2004, ASP Conf. Ser., 317, 59

Pittori, C., Verrecchia, F., Chen, A. W., et al. 2009, A\&A, 506, 1563

Tavani, M., Barbiellini, G., Argan, A., et al. 2008, Nucl. Instr. Meth. A, 588, 52

1 INAF/IASF - Milano, via E. Bassini 15, 20133 Milano, Italy e-mail: giuliani@iasf-milano.inaf.it

2 INAF/IASF - Roma, via Fosso del Cavaliere 100, 00133 Roma, Italy

3 Dip. di Fisica, Univ. "Tor Vergata", via della Ricerca Scientifica 1, 00133 Roma, Italy

4 CIFS - Torino, viale Settimio Severo 3, 10133, Torino, Italy

5 INAF/IASF - Bologna, via Gobetti 101, 40129 Bologna, Italy

6 Dip. di Fisica and INFN, via Valerio 2, 34127 Trieste, Italy

7 Department of Astrophysics, Nagoya University, Chikusa-ku, Nagoya 464-8602, Japan

8 INFN - Pavia, via Bassi 6, 27100 Pavia, Italy

9 INAF - Osservatorio Astronomico di Cagliari, localita' Poggio dei Pini, strada 54, 09012 Capoterra, Italy

10 ENEA - Bologna, via dei Martiri di Monte Sole 4, 40129 Bologna, Italy

11 INFN - Roma "La Sapienza”, Piazzale A. Moro 2, 00185 Roma, Italy

12 INFN - Roma “Tor Vergata”, via della Ricerca Scientifica 1, 00133 Roma, Italy

13 Dip. di Fisica, Univ. dell'Insubria, via Valleggio 11, 22100 Como, Italy

14 ENEA - Roma, via E. Fermi 45, 00044 Frascati (Roma), Italy

15 AS - ASDC, via G. Galilei, 00044 Frascati (Roma), Italy

16 ASI, viale Liegi 26, 00198 Roma, Italy

17 INAF/IASF - Palermo, via La Malfa 153, 90146 Palermo, Italy

18 Max - Planck - Institut für Kernphysik, 69117 Heidelberg, Germany

19 Dublin Institute for Advanced Sudies, Dublin 2, Ireland 